\title{
ON THE CARDINALITIES OF KRONECKER QUIVER GRASSMANNIANS
}

\author{
CSABA SZÁNTÓ
}

\begin{abstract}
We deduce using the Ringel-Hall algebra approach explicit formulas for the cardinalities of some Grassmannians over a finite field associated to the Kronecker quiver. We realize in this way a quantification of the formulas obtained by Caldero and Zelevinsky for the Euler characteristics of these Grassmannians. We also present a recursive algorithm for computing the cardinality of every Kronecker quiver Grassmannian over a finite field.
\end{abstract}

Key words. Kronecker algebra, Hall algebra, Grassmannian

2000 Mathematics Subject Classification. 16G20.

\section{Introduction}

Let $K$ be the Kronecker quiver $K: 1 \underset{\beta}{\longleftarrow} 2, k K$ be the Kronecker algebra over the finite field $k=\mathbb{F}_{q}$ with $q$ elements and mod- $k K$ the category of its finite dimensional right modules (called Kronecker modules). We consider the rational Ringel-Hall algebra $\mathcal{H}(k K)$ of the Kronecker algebra, with a $\mathbb{Q}$-basis formed by the isomorphism classes $[M]$ from mod- $k K$ and multiplication

$$
\left[N_{1}\right]\left[N_{2}\right]=\sum_{[M]} F_{N_{1} N_{2}}^{M}[M] .
$$

The structure constants $F_{N_{1} N_{2}}^{M}=\left|\left\{M \supseteq U \mid U \cong N_{2}, M / U \cong N_{1}\right\}\right|$ are called Ringel-Hall numbers.

For any module $M \in \bmod -k K$, and any $\underline{e}=(a, b)$ in $\mathbb{N}^{2}$, we denote by $G r_{\underline{e}}(M)_{\mathbb{F}_{q}}$ the Grassmannian of submodules of $M$ with dimension vector $\underline{e}$ :

$$
G r_{\underline{e}}(M)_{\mathbb{F}_{q}}=\{N \in \bmod -k K \mid N \leq M, \underline{\operatorname{dim}}(N)=\underline{e}\} .
$$

Then we have that

$$
\left|G r_{\underline{e}}(M)_{\mathbb{F}_{q}}\right|=\sum_{\substack{[X],[Y] \\ \underline{\operatorname{dim}} Y=\underline{e}}} F_{X Y}^{M}
$$

The Grassmannian cardinalities above play an important role in the theory of cluster algebras. In 3 . Caldero and Reineke show for affine quivers that these cardinal numbers are given by integral polynomials in $q$ with positive coefficients. So in our case there is an integral polynomial $p_{\underline{e}, M}$ such that $\left|G r_{\underline{e}}(M)_{\mathbb{F}_{q}}\right|=p_{\underline{e}, M}(q)$. Moreover the Euler characteristics $\chi\left(G r_{\underline{\underline{e}}}(M)_{\mathbb{C}}\right)=p_{\underline{e}, M}(1)$.

In 4] Caldero and Zelevinsky describe explicit combinatorial formulas for the Euler characteristics $\chi\left(G r_{\underline{e}}(M)_{\mathbb{C}}\right)=p_{\underline{e}, M}(1)$ whenever $M$ is indecomposable.

Using specific recursions obtained by the Ringel-Hall algebra approach and the use of reflection functors we deduce in this paper explicit combinatorial formulas for the cardinalities (polynomials) $\left|G r_{\underline{e}}(M)_{\mathbb{F}_{q}}\right|=p_{\underline{e}, M}(q)$ whenever $M$ is indecomposable. We obtain in this way a quantification of the formulas by Caldero and Zelevinsky. Moreover our recursions provide a 
recursive algorithm for computing the cardinality of every Kronecker quiver Grassmannian over a finite field.

\section{Facts on Kronecker modules and Ringel-Hall algebras}

The indecomposables in $\bmod -k K$ are divided into three families: the preprojectives, the regulars and the preinjectives (see [1, [2], [8]).

The preprojective (respectively preinjective) indecomposable modules are up to isomorphism uniquely determined by their dimension vectors. For $n \in \mathbb{N}$ we will denote by $P_{n}$ (respectively with $\left.I_{n}\right)$ the indecomposable preprojective module of dimension $(n+1, n)$ (respectively the indecomposable preinjective module of dimension $(n, n+1))$. So $P_{0}, P_{1}$ are the projective indecomposable modules ( $P_{0}=S_{1}$ being simple) and $I_{0}, I_{1}$ the injective indecomposable modules $\left(I_{0}=S_{2}\right.$ being simple).

The regular indecomposables (up to isomorphism) are $R_{p}(t)$ for $t \geq 1$ and $p \in \mathbb{P}_{k}^{1}$ of dimension vector $\left(t d_{p}, t d_{p}\right)\left(d_{p}\right.$ standing for the degree of the point $\left.p\right)$. The module $R_{p}(t)$ has regular length $t$ and regular socle the regular simple $R_{p}(1)$. Suppose that $R_{p}(0)=0$. Note that $R_{p}(t)$ is regular uniserial meaning that the only regular submodule series of $R_{p}(t)$ is $0 \subset R_{p}(1) \subset \ldots \subset R_{p}(t)$.

We will denote by $R_{p}(\lambda)$ (where $\lambda$ is a partition) the module $\oplus_{i} R_{p}\left(\lambda_{i}\right)$ and by $P$ (respectively $I, R)$ a module with all its indecomposable components preprojective (respectively preinjective, regular).

Denote by $c M=M \oplus \ldots \oplus M c$-times.

The following lemma is well known.

Lemma 1.1. a) $\operatorname{Hom}(R, P)=\operatorname{Hom}(I, P)=\operatorname{Hom}(I, R)=\operatorname{Ext}^{1}(P, R)=\operatorname{Ext}^{1}(P, I)=$ $\operatorname{Ext}^{1}(R, I)=0$.

b) There are no nontrivial morphisms and extensions between regular modules from different tubes, i.e. if $p \neq p^{\prime}$, then $\operatorname{Hom}\left(R_{p}(t), R_{p^{\prime}}\left(t^{\prime}\right)\right)=\operatorname{Ext}^{1}\left(R_{p}(t), R_{p^{\prime}}\left(t^{\prime}\right)\right)=0$.

c) For $n \leq m$, we have $\operatorname{dim}_{k} \operatorname{Hom}\left(P_{n}, P_{m}\right)=m-n+1$ and $\operatorname{Ext}^{1}\left(P_{n}, P_{m}\right)=0$; otherwise $\operatorname{Hom}\left(P_{n}, P_{m}\right)=0$ and $\operatorname{dim}_{k} \operatorname{Ext}^{1}\left(P_{n}, P_{m}\right)=n-m-1$. In particular $\operatorname{End}\left(P_{n}\right) \cong k$ and $\operatorname{Ext}^{1}\left(P_{n}, P_{n}\right)=0$.

d) For $n \geq m$, we have $\operatorname{dim}_{k} \operatorname{Hom}\left(I_{n}, I_{m}\right)=n-m+1$ and $\operatorname{Ext}^{1}\left(I_{n}, I_{m}\right)=0$; otherwise $\operatorname{Hom}\left(I_{n}, I_{m}\right)=0$ and $\operatorname{dim}_{k} \operatorname{Ext}^{1}\left(I_{n}, I_{m}\right)=m-n-1$. In particular $\operatorname{End}\left(I_{n}\right) \cong k$ and $\operatorname{Ext}^{1}\left(I_{n}, I_{n}\right)=0$.

e) $\operatorname{dim}_{k} \operatorname{Hom}\left(P_{n}, I_{m}\right)=n+m$ and $\operatorname{dim}_{k} \operatorname{Ext}^{1}\left(I_{m}, P_{n}\right)=m+n+2$.

f) $\operatorname{dim}_{k} \operatorname{Hom}\left(P_{n}, R_{p}(t)\right)=\operatorname{dim}_{k} \operatorname{Hom}\left(R_{p}(t), I_{n}\right)=d_{p} t$ and $\operatorname{dim}_{k} \operatorname{Ext}^{1}\left(R_{p}(t), P_{n}\right)=$ $\operatorname{dim}_{k} \operatorname{Ext}^{1}\left(I_{n}, R_{p}(t)\right)=d_{p} t$.

g) $\operatorname{dim}_{k} \operatorname{Hom}\left(R_{p}\left(t_{1}\right), R_{p}\left(t_{2}\right)\right)=\operatorname{dim}_{k} \operatorname{Ext}^{1}\left(R_{p}\left(t_{1}\right), R_{p}\left(t_{2}\right)\right)=d_{p} \min \left(t_{1}, t_{2}\right)$.

Let now $\tilde{K}$ be the quiver obtained by reversing the arrows in $K$. The category mod- $k \tilde{K}$ can be identified with the category mod- $k K$ after a formal relabeling of the vertices. In general we will denote by $\neg M \in \bmod -k \tilde{K}$ (respectively by $\neg M \in \bmod -k K$ ) the relabeled version of $M \in \bmod -k K$ (respectively of $M \in \bmod -k \tilde{K})$. So we have $\neg \neg M=M$.

For $i=1,2$ denote by mod- $k K\langle i\rangle$ (respectively by mod- $k \tilde{K}\langle i\rangle$ ) the full subcategory of modules not containing the simple $S_{i}$ (respectively the simple $\tilde{S}_{i}$ ) as a direct summand. Notice that using the formal relabeling mentioned above mod- $k \tilde{K}\langle 1\rangle$ can be identified with mod- $k K\langle 2\rangle$ and $\bmod -k \tilde{K}\langle 2\rangle$ with $\bmod -k K\langle 1\rangle$. Since the vertex 1 is a sink in the quiver $K$ and a source in $\tilde{K}$ 
the restriction of the corresponding reflection functors to the above mentioned full subcategories will give us the following inverse pair of equivalences

$$
\neg R_{1}^{+}: \bmod -k K\langle 1\rangle \rightarrow \bmod -k K\langle 2\rangle \text { and } R_{1}^{-} \neg: \bmod -k K\langle 2\rangle \rightarrow \bmod -k K\langle 1\rangle .
$$

We refer to [5] for all notions and properties related to the reflection functors. Notice that we have

$$
\neg R_{1}^{+}\left(P_{n}\right)=P_{n-1} \text { for } n \in \mathbb{N}^{*}, \neg R_{1}^{+}\left(I_{n}\right)=I_{n+1} \text { for } n \in \mathbb{N} .
$$

Moreover for each $d$ there is a permutation $\sigma_{d}$ of the set $\left\{p \in \mathbb{P}_{k}^{1} \mid d_{p}=d\right\}$ such that for each $p$ with $d_{p}=d$ we have

$$
\neg R_{1}^{+}\left(R_{p}(t)\right)=R_{\sigma_{d}(p)}(t) \text { for all } t \in \mathbb{N}^{*} .
$$

Remark 1.2. Notice that if $M \in \bmod -k K\langle 1\rangle$ with $\underline{\operatorname{dim}} M=(m, n)$, then $m-n$ is at most $n$ and $\underline{\operatorname{dim}}\left(\neg R_{1}^{+}(M)\right)=(m-(m-n), n-(m-n))=(n, 2 n-m)$. Also if $M \in \bmod -k K\langle 2\rangle$ with $\underline{\operatorname{dim}} M=(m, n)$, then $n-m$ is at most $m$ and $\underline{\operatorname{dim}}\left(R_{1}^{-} \neg(M)\right)=(m+(m-n), n+(m-n))=$ $(2 m-n, m)$.

Related with the Ringel-Hall algebra we will need the following properties (see [9]):

Lemma 1.3. (Associativity of the Ringel-Hall multiplication) $\sum_{[X]} F_{A B}^{X} F_{X C}^{M}=\sum_{[X]} F_{A X}^{M} F_{B C}^{X}$.

Lemma 1.4. For $N_{1}, N_{2} \in \bmod -k K$ with $\operatorname{Ext}^{1}\left(N_{1}, N_{2}\right)=0$ and $\operatorname{Hom}\left(N_{2}, N_{1}\right)=0$ we have $\left[N_{1}\right]\left[N_{2}\right]=\left[N_{1} \oplus N_{2}\right]$.

Lemma 1.5. a) If $M, N$ and $L$ are in $\bmod -k K\langle 1\rangle$, then $F_{M N}^{L}=F_{\neg R_{1}^{+}(M) \neg R_{1}^{+}(N)}^{\neg R^{+}(L)}$.

b) If $M, N$ and $L$ are in $\bmod -k K\langle 2\rangle$, then $F_{M N}^{L}=F_{R_{1}^{-} \neg(M) R_{1}^{-} \neg(N)}^{R^{-} \neg(L)}$.

\section{Identities for Gaussian coefficients}

For $l, a \in \mathbb{Z}, l>0$ we will denote by $G_{a}^{l}(q)=\frac{\left(q^{a}-1\right) \ldots\left(q^{a-l+1}-1\right)}{\left(q^{l}-1\right) \ldots(q-1)}$ the Gaussian (q-binomial) coefficients. By definition $G_{a}^{0}(q)=1$ and $G_{a}^{-l}(q)=0$. The following properties of the Gaussian coefficients are well known

Lemma 2.1. a) $G_{a}^{l}(q)=0$ for $0 \leq a<l$. Also $G_{a}^{l}=G_{a}^{a-l}$ for $a, l \geq 0$.

b) (Cross product) For all $a, l, j \in \mathbb{Z}$ we have $G_{a}^{l}(q) G_{l}^{j}(q)=G_{a}^{j}(q) G_{a-j}^{l-j}(q)$.

c) (q-Vandermonde) For all $l, a, b \in \mathbb{Z}$ we have $G_{a+b}^{l}(q)=\sum_{j \in \mathbb{Z}} q^{j(a-l+j)} G_{a}^{l-j}(q) G_{b}^{j}(q)=$ $\sum_{r \in \mathbb{Z}} q^{(l-r)(a-r)} G_{a}^{r}(q) G_{b}^{l-r}(q)$. Notice that the sums are finite.

Finally we will prove a q-analogue of the so called Nanjundiah identity (see [7])

Proposition 2.2. For all $m, p, \mu, \nu \in \mathbb{Z}$ we have

$$
\sum_{r \in \mathbb{Z}} q^{(m-\mu+\nu-r)(p-r)} G_{m-\mu+\nu}^{r}(q) G_{p+\mu-\nu}^{p-r}(q) G_{\mu+r}^{m+p}(q)=G_{\mu}^{m}(q) G_{\nu}^{p}(q)
$$

Proof. Denote by $A$ the left expression and by $B$ the right one.

One can immediately see that for $p<0$ we have $A=B=0$.

Applying 3 times Lemma 2.1. c) and 2 times Lemma 2.1. b) we have

$$
A=\sum_{r \in \mathbb{Z}} q^{(m-\mu+\nu-r)(p-r)} G_{m-\mu+\nu}^{r}(q) G_{p+\mu-\nu}^{p-r}(q) G_{\mu+r}^{m+p}(q)
$$




$$
\begin{gathered}
=\sum_{r} q^{(m-\mu+\nu-r)(p-r)} G_{m-\mu+\nu}^{r}(q) G_{p+\mu-\nu}^{p-r}(q) \sum_{s \in \mathbb{Z}} q^{s(\mu-m-p+s)} G_{\mu}^{m+p-s}(q) G_{r}^{s}(q) \\
=\sum_{r, s \in \mathbb{Z}} q^{s(\mu-m-p+s)} q^{(m-\mu+\nu-r)(p-r)} G_{m-\mu+\nu}^{r}(q) G_{r}^{s}(q) G_{p+\mu-\nu}^{p-r}(q) G_{\mu}^{m+p-s}(q) \\
=\sum_{r, s \in \mathbb{Z}} q^{s(\mu-m-p+s)} q^{(m-\mu+\nu-r)(p-r)} G_{m-\mu+\nu}^{s}(q) G_{m-\mu+\nu-s}^{r-s}(q) G_{p+\mu-\nu}^{p-r}(q) G_{\mu}^{m+p-s}(q) \\
=\sum_{s \in \mathbb{Z}} q^{s(\mu-m-p+s)} G_{m-\mu+\nu}^{s}(q) G_{\mu}^{m+p-s}(q) \sum_{r \in \mathbb{Z}} q^{(m-\mu+\nu-r)(p-r)} G_{m-\mu+\nu-s}^{r-s}(q) G_{p+\mu-\nu}^{p-r}(q) \\
=\sum_{s \in \mathbb{Z}} q^{s(\mu-m-p+s)} G_{m-\mu+\nu}^{s}(q) G_{\mu}^{m+p-s}(q) \sum_{t \in \mathbb{Z}} q^{(m-\mu+\nu-s-t)(p-s-t)} G_{m-\mu+\nu-s}^{t}(q) G_{p+\mu-\nu}^{p-s-t}(q) \\
=\sum_{s \in \mathbb{Z}} q^{s(\mu-m-p+s)} G_{m-\mu+\nu}^{s}(q) G_{\mu}^{m+p-s}(q) G_{m+p-s}^{p-s}(q) \\
=\sum_{s \in \mathbb{Z}} q^{s(\mu-m-p+s)} G_{m-\mu+\nu}^{s}(q) G_{\mu}^{p-s}(q) G_{\mu-p+s}^{m}(q)
\end{gathered}
$$

One can see from here that for $m<0$ we have $A=0$ and trivially also $B=0$. Consider now the case $m, p \geq 0$. Then using Lemma 2.1. a),b) notice that

$$
\begin{gathered}
A=\sum_{s \in \mathbb{Z}} q^{s(\mu-m-p+s)} G_{m-\mu+\nu}^{s}(q) G_{\mu}^{m+p-s}(q) G_{m+p-s}^{p-s}(q) \\
=\sum_{s=0}^{p} q^{s(\mu-m-p+s)} G_{m-\mu+\nu}^{s}(q) G_{\mu}^{m+p-s}(q) G_{m+p-s}^{p-s}(q) \\
=\sum_{s=0}^{p} q^{s(\mu-m-p+s)} G_{m-\mu+\nu}^{s}(q) G_{\mu}^{m+p-s}(q) G_{m+p-s}^{m}(q) \\
=\sum_{s \in \mathbb{Z}} q^{s(\mu-m-p+s)} G_{m-\mu+\nu}^{s}(q) G_{\mu}^{m+p-s}(q) G_{m+p-s}^{m}(q) \\
=\sum_{s \in \mathbb{Z}} q^{s(\mu-m-p+s)} G_{m-\mu+\nu}^{s}(q) G_{\mu}^{m}(q) G_{\mu-m}^{p-s}(q) \\
=G_{\mu}^{m}(q) \sum_{s \in \mathbb{Z}} q^{s(\mu-m-p+s)} G_{m-\mu+\nu}^{s}(q) G_{\mu-m}^{p-s}(q) \\
=G_{\mu}^{m}(q) G_{\nu}^{p}(q)=B
\end{gathered}
$$

\section{The recursions}

Let $a, b \in \mathbb{Z}$. We introduce the following notations.

For $M \in \bmod -k K$

$$
A_{a, b}^{M}:=\left|G r_{(a, b)}(M)\right|=\sum_{\substack{[X],[Y] \\ \underline{\operatorname{dim} Y}=(a, b)}} F_{X Y}^{M}
$$

For $M \in \bmod -k K\langle 1\rangle$

$$
B_{a, b}^{M}:=\sum_{\substack{[X],[Y] \\ \text { dim } Y=(a, b) \\ X, Y \in \bmod -k K\langle 1\rangle}} F_{X Y}^{M}
$$


For $M \in \bmod -k K\langle 2\rangle$

$$
C_{a, b}^{M}:=\sum_{\substack{[X],[Y] \\ \text { dim } Y=(a, b) \\ Y, \bmod -k K\langle 2\rangle}} F_{X Y}^{M}
$$

The sums $A_{a, b}^{M}, B_{a, b}^{M}, C_{a, b}^{M}$ are considered to be 0 if they are empty. In particular they are 0 if $a<0$ or $b<0$. Also notice that if $\underline{\operatorname{dim}} M=(m, n)$ then $A_{a, b}^{M}=B_{a, b}^{M}=C_{a, b}^{M}=0$ for $a>m$ or $b>n$.

Proposition 3.1. Suppose up to isomorphism $M=s S_{1} \oplus M^{\prime} \oplus t S_{2}$ with $M^{\prime} \in \bmod -k K\langle 1\rangle \cap$ $\bmod -k K\langle 2\rangle$. Let $a, b \in \mathbb{Z}, l=a-b$ and $\underline{\operatorname{dim}} M=(m, n)$.

a) We have that

$$
A_{a, b}^{M}=\sum_{c \in \mathbb{Z}} G_{m-a+c}^{c}(q) B_{a-c, b}^{M^{\prime} \oplus t S_{2}}=\sum_{c \in \mathbb{Z}} G_{m-a+c}^{c}(q) C_{a-l, b-l+c}^{\neg R_{1}^{+}\left(M^{\prime} \oplus t S_{2}\right)},
$$

the sum being finite.

b) We have that

$$
A_{a, b}^{M}=\sum_{d \in \mathbb{Z}} G_{b+d}^{d}(q) C_{a, b+d}^{s S_{1} \oplus M^{\prime}}=\sum_{d \in \mathbb{Z}} G_{b+d}^{d}(q) B_{a+l-d, b+l}^{R_{1}^{-} \neg\left(s S_{1} \oplus M^{\prime}\right)},
$$

the sum being finite.

Proof. a) If $b<0$ then trivially

$$
A_{a, b}^{M}=\sum_{c \in \mathbb{Z}} G_{m-a+c}^{c}(q) B_{a-c, b}^{M^{\prime} \oplus t S_{2}}=\sum_{c \in \mathbb{Z}} G_{m-a+c}^{c}(q) C_{b, b-l+c}^{\neg R_{1}^{+}\left(M^{\prime} \oplus t S_{2}\right)}=0 .
$$

If $a<0$ then $A_{a, b}^{M}=0$. The sum $\sum_{c \in \mathbb{Z}} G_{m-a+c}^{c}(q) B_{a-c, b}^{M^{\prime} \oplus t S_{2}}=0$ because for $c<0 G_{m-a+c}^{c}(q)=0$ and for $c \geq 0$ we have $a-c<0$ so $B_{a-c, b}^{M^{\prime} \oplus t S_{2}}=0$. We also have $\sum_{c \in \mathbb{Z}} G_{m-a+c}^{c}(q) C_{b, 2 b-a+c}^{\neg R_{1}^{+}\left(M^{\prime} \oplus t S_{2}\right)}=0$ because for $c<0 G_{m-a+c}^{c}(q)=0$ and for $c \geq 0$ there is no $Y \in \bmod -k K\langle 2\rangle$ with dimension $(b, 2 b-a+c)$ (see Remark 1.2.) so $C_{a-l, b-l+c}^{\neg R_{1}^{+}\left(M^{\prime} \oplus t S_{2}\right)}=0$.

Consider now the case $a, b \geq 0$. Firstly notice that if $Y_{c} \in \bmod -k K\langle 1\rangle$ then by Lemma 1.1. and Lemma 1.4. we have $\left[c S_{1}\right]\left[Y_{c}\right]=\left[c S_{1} \oplus Y_{c}\right]$, so $F_{c S_{1} Y_{c}}^{Z}=1$ for $[Z]=\left[c S_{1} \oplus Y_{c}\right]$ and $F_{c S_{1} Y_{c}}^{Z}=0$ in all the other cases.

Using Lemma 1.3. we obtain:

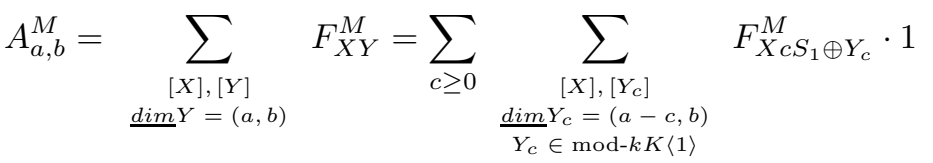

$$
\begin{aligned}
& =\sum_{c \geq 0} \sum_{\begin{array}{c}
{[X],\left[Y_{c}\right],[Z]} \\
\text { dim } Y_{c}=(a-c, b) \\
Y_{c} \in \bmod -k K\langle 1\rangle
\end{array}} F_{X Z}^{M} \cdot F_{c S_{1} Y_{c}}^{Z}=\sum_{c \geq 0} \sum_{\begin{array}{c}
{[X],\left[Y_{c}\right],[Z]} \\
\text { dim } Y_{c}=(a-c, b) \\
Y_{c} \in \bmod -k K\langle 1\rangle
\end{array}} F_{X c S_{1}}^{Z} \cdot F_{Z Y_{c}}^{M}
\end{aligned}
$$

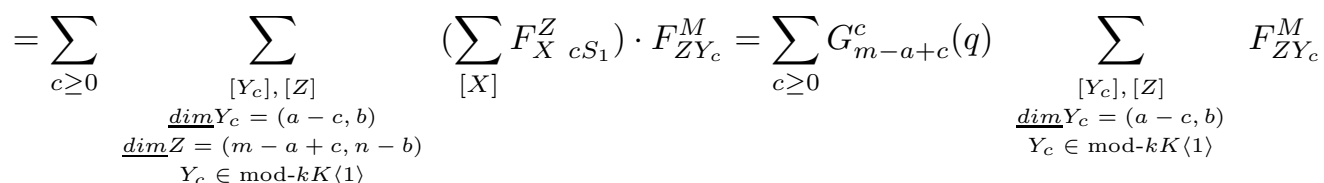




$$
=\sum_{c \geq 0} G_{m-a+c}^{c}(q) \sum_{\substack{\left[Y_{c}\right],\left[Z^{\prime}\right] \\ \text { dim } Y_{c}=(a-c, b) \\ Y_{c}, Z^{\prime} \in \bmod -k K\langle 1\rangle}} F_{Z^{\prime} Y_{c}}^{M^{\prime} \oplus t S_{2}}=\sum_{c \in \mathbb{Z}} G_{m-a+c}^{c}(q) B_{a-c, b}^{M^{\prime} \oplus t S_{2}}
$$

Here we have used the following: if $F_{Z Y_{c}}^{M}=F_{Z Y_{c}}^{s S_{1} \oplus M^{\prime} \oplus t S_{2}} \neq 0$ then since $Y_{c} \in \bmod -k K\langle 1\rangle$ it follows that $Y_{c}$ embeds only in $M^{\prime} \oplus t S_{2}$ (see Lemma 1.1.), so $Z=s S_{1} \oplus Z^{\prime}$ with $0 \rightarrow Y_{c} \rightarrow$ $M^{\prime} \oplus t S_{2} \rightarrow Z^{\prime} \rightarrow 0$ exact, $Z^{\prime} \in \bmod -k K\langle 1\rangle$ (because $M^{\prime} \oplus t S_{2}$ does not project on $S_{1}$ ) and in this way $F_{Z Y_{c}}^{s S_{1} \oplus M^{\prime} \oplus t S_{2}}=F_{Z^{\prime} Y_{c}}^{M^{\prime} \oplus t S_{2}}$.

To prove the other identity we will use reflection functors. Using Remark 1.2. and Lemma 1.5. we have

$$
\begin{aligned}
& A_{a, b}^{M}=\sum_{c \geq 0} G_{m-a+c}^{c}(q) \sum_{\substack{\left[Y_{c}\right],\left[Z^{\prime}\right] \\
\operatorname{dim} Y_{c}=(a-c, b)}} F_{Z^{\prime} Y_{c}}^{M^{\prime} \oplus t S_{2}} \\
& \begin{array}{c}
\frac{\operatorname{dim}}{Y_{c}} Y_{c}=(a-c, b) \\
Y_{c}, Z^{\prime} \in \bmod -k K\langle 1\rangle
\end{array} \\
& =\sum_{c \geq 0} G_{m-a+c}^{c}(q) \quad \sum_{\left[Y_{c}\right],\left[Z^{\prime}\right]} \quad F_{\neg R_{1}^{+}\left(Z^{\prime}\right) \neg R_{1}^{+}\left(Y_{c}\right)}^{\neg R^{+}\left(M^{\prime} \oplus t S_{2}\right)} \\
& \begin{array}{c}
\frac{\operatorname{dim} Y_{c}=(a-c, b)}{Y_{c}, Z^{\prime} \in \bmod -k K\langle 1\rangle}
\end{array} \\
& =\sum_{c \geq 0} G_{m-a+c}^{c}(q) \sum_{\substack{\operatorname{dim} Y_{c}^{\prime}=\left(Y_{c}^{\prime}\right],\left[Z^{\prime \prime}\right] \\
Y_{c}^{\prime}, Z^{\prime \prime} \in \bmod -k K\langle 2\rangle}} F_{Z^{\prime \prime} Y_{c}^{\prime}}^{\neg R^{+}\left(M^{\prime} \oplus t S_{2}\right)} \\
& =\sum_{c \in \mathbb{Z}} G_{m-a+c}^{c}(q) C_{a-l, b-l+c}^{\neg R_{1}^{+}\left(M^{\prime} \oplus t S_{2}\right)} .
\end{aligned}
$$

b) dual of a).

We can state now the recursion theorem for the numbers $A_{a, b}^{M}$.

Theorem 3.2. Suppose up to isomorphism $M=s S_{1} \oplus M^{\prime} \oplus t S_{2}$ with $M^{\prime} \in \bmod -k K\langle 1\rangle \cap$ $\bmod -k K\langle 2\rangle$. Let $a, b \in \mathbb{Z}, l=a-b$ and $\underline{\operatorname{dim}} M=(m, n)$. We have the following recursions

a)

$$
A_{a, b}^{M}=\sum_{c \in \mathbb{Z}} q^{c(b-l+c)} G_{m-2 b}^{c}(q) A_{a-l, b-l+c}^{\neg R_{1}^{+}\left(M^{\prime} \oplus t S_{2}\right)}
$$

the sum being finite.

b)

$$
A_{a, b}^{M}=\sum_{d \in \mathbb{Z}} q^{d(2 m-n+t-a-l+d)} G_{2 a-2 m+n-t}^{d}(q) A_{a+l-d, b+l}^{R_{1}^{-} \neg\left(s S_{1} \oplus M^{\prime}\right)},
$$

the sum being finite.

Proof. a) Using the previous proposition and the fact that $\neg R_{1}^{+}\left(M^{\prime} \oplus t S_{2}\right) \in \bmod -k K\langle 2\rangle$ we have

$$
\begin{gathered}
A_{a, b}^{M}=\sum_{c \in \mathbb{Z}} G_{m-a+c}^{c}(q) C_{a-l, b-l+c}^{\neg R_{1}^{+}\left(M^{\prime} \oplus t S_{2}\right)}, \\
A_{a-l, b-l+c}^{\neg R_{1}^{+}\left(M^{\prime} \oplus t S_{2}\right)}=\sum_{d \in \mathbb{Z}} G_{b-l+c+d}^{d}(q) C_{a-l, b-l+c+d}^{\neg R_{1}^{+}\left(M^{\prime} \oplus t S_{2}\right)} .
\end{gathered}
$$

Let $u=c+d$. Using Lemma 2.1. c)

$$
\sum_{c \in \mathbb{Z}} q^{c(b-l+c)} G_{m-2 b}^{c}(q) A_{a-l, b-l+c}^{\neg R_{1}^{+}\left(M^{\prime} \oplus t S_{2}\right)}=\sum_{c, d \in \mathbb{Z}} q^{c(b-l+c)} G_{m-2 b}^{c}(q) G_{b-l+c+d}^{d}(q) C_{a-l, b-l+c+d}^{\neg R_{1}^{+}\left(M^{\prime} \oplus t S_{2}\right)}
$$


$=\sum_{u \in \mathbb{Z}}\left(\sum_{c \in \mathbb{Z}} q^{c(b-l+c)} G_{m-2 b}^{c}(q) G_{b-l+u}^{u-c}(q)\right) C_{a-l, b-l+u}^{\neg R_{1}^{+}\left(M^{\prime} \oplus t S_{2}\right)}=\sum_{u \in \mathbb{Z}} G_{m-a+u}^{u}(q) C_{a-l, b-l+u}^{\neg R_{1}^{+}\left(M^{\prime} \oplus t S_{2}\right)}=A_{a, b}^{M}$.

b) Using the previous proposition, Remark 1.2. and the fact that $R_{1}^{-} \neg\left(s S_{1} \oplus M^{\prime}\right) \in \bmod -k K\langle 1\rangle$ we have

$$
\begin{gathered}
A_{a, b}^{M}=\sum_{d \in \mathbb{Z}} G_{b+d}^{d}(q) B_{a+l-d, b+l}^{R_{1}^{-} \neg\left(s S_{1} \oplus M^{\prime}\right)}, \\
A_{a+l-d, b+l}^{R_{1}^{-} \neg\left(s S_{1} \oplus M^{\prime}\right)}=\sum_{c \in \mathbb{Z}} G_{2 m-n+t-a-l+d+c}^{c}(q) B_{a+l-d-c, b+l}^{R_{1}^{-} \neg\left(s S_{1} \oplus M^{\prime}\right)} .
\end{gathered}
$$

Let $u=c+d$. Using Lemma 2.1. c)

$$
\begin{gathered}
\sum_{d \in \mathbb{Z}} q^{d(2 m-n+t-a-l+d)} G_{2 a-2 m+n-t}^{d}(q) A_{a+l-d, b+l}^{R_{1}^{-} \neg\left(s S_{1} \oplus M^{\prime}\right)} \\
=\sum_{c, d \in \mathbb{Z}} q^{d(2 m-n+t-a-l+d)} G_{2 a-2 m+n-t}^{d}(q) G_{2 m-n+t-a-l+d+c}^{c}(q) B_{a+l-d-c, b+l}^{R_{1}^{-} \neg\left(s S_{1} \oplus M^{\prime}\right)} \\
=\sum_{u \in \mathbb{Z}}\left(\sum_{d \in \mathbb{Z}} q^{d(2 m-n+t-a-l+d)} G_{2 a-2 m+n-t}^{d}(q) G_{2 m-n+t-a-l+u}^{u-d}(q)\right) B_{a+l-u, b+l}^{\left.R_{1}^{-}\right\urcorner\left(s S_{1} \oplus M^{\prime}\right)} \\
=\sum_{u \in \mathbb{Z}} G_{b+u}^{u}(q) B_{a+l-u, b+l}^{R_{1}^{-} \neg\left(s S_{1} \oplus M^{\prime}\right)}=A_{a, b}^{M} .
\end{gathered}
$$

\section{Formulas for the cardinalities $A_{a, b}^{M}=\left|G r_{(a, b)}(M)\right|$ with $M$ indecomposable}

Using the recurrences from the previous section we will provide closed formulas for $A_{a, b}^{P_{n}}, A_{a, b}^{I_{n}}$ (with $n \in \mathbb{N}, a, b \in \mathbb{Z}$ ) and $A_{a, b}^{R_{p}(t)}$ (with $t \in \mathbb{N}^{*}, a, b \in \mathbb{Z}$ and $p \in \mathbb{P}_{k}^{1}$ of degree 1 ).

Theorem 4.1. $A_{a, b}^{P_{n}}=\left|G r_{(a, b)}\left(P_{n}\right)\right|=\left\{\begin{array}{cc}0 & \text { for } a<0 \text { or } b<0 \\ 1 & \text { for } a=b=0 \\ G_{n+1-b}^{n+1-a}(q) G_{a-1}^{a-b-1}(q) & \text { otherwise }\end{array}\right.$

Remark 4.2. Using the definitions and Lemma 2.1. a) notice that $G_{n+1-b}^{n+1-a}(q) G_{a-1}^{a-b-1}=0$ for $0<a \leq b$, for $a>n+1$, for $b>n$, for $a>0$ and $b<0$.

Proof. Induction on $n$. For $n=0$ we have that $A_{a, b}^{P_{0}}=1$ when $(a, b)=(1,0)$ or $(a, b)=(0,0)$ and 0 otherwise so using the previous remark we can see that the formula is true.

Suppose now $n \geq 1$. Then trivially $A_{a, b}^{P_{n}}=0$ for $a<0$ or $b<0$ and $A_{0,0}^{P_{n}}=1$ so we only need to look at the case $a, b \geq 0, a^{2}+b^{2} \neq 0$. Using Theorem 3.2. a) we obtain the recursion

$$
A_{a, b}^{P_{n}}=\sum_{c \in \mathbb{Z}} q^{c(b-l+c)} G_{n-2 b+1}^{c}(q) A_{a-l, b-l+c}^{P_{n-1}},
$$

the sum being finite.

Using Remark 4.2. and the induction hypothesis notice that if $b>0$ (and $a \geq 0$ ) then

$$
A_{a-l, b-l+c}^{P_{n-1}}=A_{b, 2 b-a+c}^{P_{n-1}}=G_{n-2 b+a-c}^{n-b}(q) G_{b-1}^{a-b-c-1}(q)
$$

so denoting by $u=a-b-c-1$, using the previous recursion and Proposition 2.2. with the entries $p=a-b-1, m=n+1-a, \mu=n+1-b$ and $\nu=a-1$

$$
A_{a, b}^{P_{n}}=\sum_{c \in \mathbb{Z}} q^{c(b-l+c)} G_{n-2 b+1}^{c}(q) A_{a-l, b-l+c}^{P_{n-1}}
$$




$$
\begin{gathered}
=\sum_{c \in \mathbb{Z}} q^{c(2 b-a+c)} G_{n-2 b+1}^{c}(q) G_{n-2 b+a-c}^{n-b}(q) G_{b-1}^{a-b-c-1}(q) \\
=\sum_{u \in \mathbb{Z}} q^{(a-b-u-1)(b-u-1)} G_{n-2 b+1}^{a-b-u-1}(q) G_{n-b+1+u}^{n-b}(q) G_{b-1}^{u}(q)=G_{n+1-b}^{n+1-a}(q) G_{a-1}^{a-b-1}(q)
\end{gathered}
$$

If now $b=0$ (and $n+1 \geq a>0$ ) then trivially

$$
A_{a, b}^{P_{n}}=\sum_{[X]} F_{X{ }^{\prime} S_{1}}^{P_{n}}=G_{n+1}^{a}(q)=G_{n+1-0}^{n+1-a}(q) G_{a-1}^{a-0-1}(q) .
$$

If $b=0$ and $a>n+1$ then trivially $A_{a, b}^{P_{n}}=0$ (see Remark 4.2.).

Theorem 4.3. $A_{a, b}^{I_{n}}=\left|G r_{(a, b)}\left(I_{n}\right)\right|=\left\{\begin{array}{cc}0 & \text { for } a>n \text { or } b>n+1 \\ 1 & \text { for } a=n, b=n+1 \\ G_{n-b}^{a-b}(q) G_{a+1}^{b}(q) & \text { otherwise }\end{array}\right.$

Remark 4.4. Using the definitions and Lemma 2.1. a) notice that $G_{n-b}^{a-b}(q) G_{a+1}^{b}=0$ for $a<b$, for $a<0$, for $b<0$, for $a>n$ and $b<n+1$.

Proof. Induction on $n$. For $n=0$ we have that $A_{a, b}^{I_{0}}=1$ when $(a, b)=(0,1)$ or $(a, b)=(0,0)$ and 0 otherwise so using the previous remark we can see that the formula is true.

Suppose now $n \geq 1$. Then trivially $A_{a, b}^{I_{n}}=0$ for $a>n$ or $b>n+1$ and $A_{n, n+1}^{I_{n}}=1$ so we only need to look at the case $a \leq n, b \leq n+1$ (with no simultaneous equality). Using Theorem 3.2 . b) we obtain the recursion

$$
A_{a, b}^{I_{n}}=\sum_{d \in \mathbb{Z}} q^{d(n-1-a-l+d)} G_{2 a-n+1}^{d}(q) A_{a+l-d, b+l}^{I_{n-1}},
$$

the sum being finite.

Using Remark 4.4. and the induction hypothesis notice that if $a<n($ and $b \leq n+1)$ then

$$
A_{a+l-d, b+l}^{I_{n-1}}=A_{2 a-b-d, a}^{I_{n-1}}=G_{n-a-1}^{a-b-d}(q) G_{2 a-b-d+1}^{a}(q)
$$

so denoting by $u=a-b-d$, using the previous recursion and Proposition 2.2. with the entries $p=a-b, m=b, \mu=a+1$ and $\nu=n-b$

$$
\begin{gathered}
A_{a, b}^{I_{n}}=\sum_{d \in \mathbb{Z}} q^{d(n-1-a-l+d)} G_{2 a-n+1}^{d}(q) A_{a+l-d, b+l}^{I_{n-1}} \\
=\sum_{d \in \mathbb{Z}} q^{d(n-1-2 a+b+d)} G_{2 a-n+1}^{d}(q) G_{n-a-1}^{a-b-d}(q) G_{2 a-b-d+1}^{a}(q) \\
=\sum_{u \in \mathbb{Z}} q^{(a-b-u)(n-1-a-u)} G_{2 a-n+1}^{a-b-u}(q) G_{n-a-1}^{u}(q) G_{a+u+1}^{a}(q)=G_{n-b}^{a-b}(q) G_{a+1}^{b}(q) .
\end{gathered}
$$

If now $a=n$ (and $0 \leq b<n+1$ ) then trivially

$$
A_{a, b}^{I_{n}}=\sum_{[X]} F_{(n+1-b) S_{2} X}^{I_{n}}=G_{n+1}^{n+1-b}(q)=G_{n-b}^{n-b}(q) G_{n+1}^{b}(q) .
$$

If $a=n$ and $b<0$ then trivially $A_{a, b}^{I_{n}}=0$ (see Remark 4.4.).

Lemma 4.5. Let $t \in \mathbb{N}^{*}, a, b \in \mathbb{Z}$ and $p \in \mathbb{P}_{k}^{1}$ of degree 1 . Then we have

a) $A_{a, a}^{R_{p}(t)}=1$ for $0 \leq a \leq t$.

b) $A_{a, b}^{R_{p}(t)}=0$ for $0 \leq a<b \leq t$.

c) For two points $p, p^{\prime} \in \mathbb{P}_{k}^{1}$ of the same degree 1 we have, that $A_{a, b}^{R_{p}(t)}=A_{a, b}^{R_{p^{\prime}}(t)}$. 
Proof. a) Suppose $\underline{\operatorname{dim}} Y=(a, a), a>0$ (so the defect is 0 ) and $Y$ embeds into $R_{p}(t)$. Then using Lemma 1.1. and the uniseriality of the regulars one can see that $Y$ must be of the form $R_{p}\left(t^{\prime}\right)$ with $0<t^{\prime} \leq t$. So it follows that for $0<a \leq n$, we have $A_{a, a}^{R_{p}(t)}=F_{R_{p}\left(t^{\prime \prime}\right) R_{p}\left(t^{\prime}\right)}^{R_{p}(t)}=1$. The rest of the statement follows easily.

b) If for $0 \leq a<b \leq t A_{a, b}^{R_{p}(t)}>0$ this would mean that there is a module $Y$ of dimension $(a, b)$ which embeds into $R_{p}(t)$. But $a<b$ means that $Y$ must have a preinjective component. Using Lemma 1.1. one can notice that we can't embed a preinjective into a regular module.

c) Using Lemma 1.1. and the uniseriality of regulars, observe that for $F_{X Y}^{R_{p}(t)} \neq 0$ the modules $X, Y$ can contain at most a single regular direct component which is of the form $R_{p}\left(t^{\prime}\right)$. Permuting the points $\left\{p \in \mathbb{P}_{k}^{1} \mid d_{p}=d\right\}$ the assertion follows.

Theorem 4.6. Let $t \in \mathbb{N}^{*}, a, b \in \mathbb{Z}$ and $p \in \mathbb{P}_{k}^{1}$ of degree 1 . Then we have

$$
A_{a, b}^{R_{p}(t)}=\left|G r_{(a, b)}\left(R_{p}(t)\right)\right|=\left\{\begin{array}{cc}
0 & \text { for } a<0 \text { or } b<0 \\
G_{t-b}^{t-a}(q) G_{a}^{a-b}(q) & \text { otherwise }
\end{array}\right.
$$

Remark 4.7. Using the definitions and Lemma 2.1. a) notice that $G_{t-b}^{t-a}(q) G_{a}^{a-b}=0$ for $a<b$, for $a>t$, for $b>t$ and $G_{t-b}^{t-a}(q) G_{a}^{a-b}=1$ for $0 \leq a=b \leq t$.

Proof. Using Remark 4.7. observe that the formula is trivially true whenever $a<0$ or $b<0$ or $a>t$ or $b>t$. Also when $b=0$ and $0 \leq a \leq t$ then trivially

$$
A_{a, 0}^{R_{p}(t)}=\sum_{[X]} F_{X a S_{1}}^{R_{p}(t)}=G_{t}^{a}(q)=G_{t-0}^{t-a}(q) G_{a}^{a-0}(q) .
$$

Using Lemma 4.5. one can see that the formula is true in the cases $0 \leq a=b \leq t$ and $0 \leq a<b \leq t$.

So we only need to consider the case $0<b<a \leq t$. Using Theorem 3.2. a) and Lemma 4.5. c) we obtain the recursion

$$
A_{a, b}^{R_{p}(t)}=\sum_{c \in \mathbb{Z}} q^{c(b-l+c)} G_{t-2 b}^{c}(q) A_{a-l, b-l+c}^{R_{p}(t)},
$$

the sum being finite.

We proceed by induction on $a$. Using the recursion and the considerations above for $a=2 \leq t$ we have

$$
A_{2,1}^{R_{p}(t)}=\sum_{c \in \mathbb{Z}} q^{c^{2}} G_{t-2}^{c}(q) A_{1, c}^{R_{p}(t)}=G_{t-2}^{0} G_{t}^{1}+q G_{t-2}^{1}=G_{t-1}^{t-2} G_{2}^{1}
$$

Let now $3 \leq a \leq t$ and $0<b<a$. Using Remark 4.7. and the induction hypothesis notice that

$$
A_{a-l, b-l+c}^{R_{p}(t)}=A_{b, 2 b-a+c}^{R_{p}(t)}=G_{t-2 b+a-c}^{t-b}(q) G_{b}^{a-b-c}(q)
$$

so denoting by $u=a-b-c$, using the previous recursion and Proposition 2.2. with the entries $p=a-b, m=t-a, \mu=t-b$ and $\nu=a$

$$
\begin{gathered}
A_{a, b}^{R_{p}(t)}=\sum_{c \in \mathbb{Z}} q^{c(b-l+c)} G_{t-2 b}^{c}(q) A_{a-l, b-l+c}^{R_{p}(t)} \\
=\sum_{c \in \mathbb{Z}} q^{c(2 b-a+c)} G_{t-2 b}^{c}(q) G_{t-2 b+a-c}^{t-b}(q) G_{b}^{a-b-c}(q) \\
=\sum_{u \in \mathbb{Z}} q^{(a-b-u)(b-u)} G_{t-2 b}^{a-b-u}(q) G_{t-b+u}^{t-b}(q) G_{b}^{u}(q)=G_{t-b}^{t-a}(q) G_{a}^{a-b}
\end{gathered}
$$


We can see that in the cases above $\left|G r_{\underline{e}}(M)_{\mathbb{F}_{q}}\right|$ is an integer polynomial $p_{\underline{e}}, M(q)$. Using that $\chi\left(G r_{\underline{e}}(M)_{\mathbb{C}}\right)=p_{\underline{e}, M}(1)$ and $G_{a}^{n}(1)=\left(\begin{array}{l}a \\ n\end{array}\right)$ we obtain

Corollary 4.8. 4

$$
\begin{aligned}
& \text { a) } \chi\left(G r_{(a, b)}\left(P_{n}\right)_{\mathbb{C}}\right)=\left\{\begin{array}{cc}
0 & \text { for } a<0 \text { or } b<0 \\
1 & \text { for } a=b=0 \\
\left(\begin{array}{l}
n+1-b \\
n+1-a
\end{array}\right)\left(\begin{array}{c}
a-1 \\
a-b-1
\end{array}\right) & \text { otherwise }
\end{array}\right. \\
& \text { b) } \chi\left(G r_{(a, b)}\left(I_{n}\right)_{\mathbb{C}}\right)=\left\{\begin{array}{cc}
0 & \text { for } a>n \text { or } b>n+1 \\
1 & \text { for } a=n, b=n+1 \\
\left(\begin{array}{l}
n-b \\
a-b
\end{array}\right)\left(\begin{array}{c}
a+1 \\
b
\end{array}\right) & \text { otherwise }
\end{array}\right.
\end{aligned}
$$

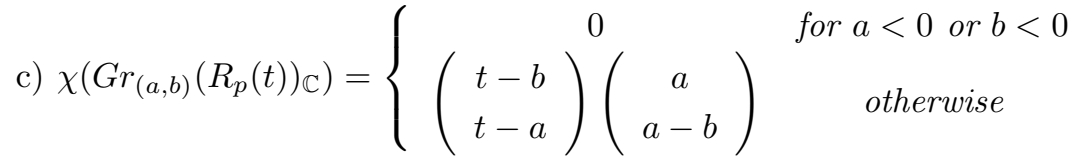

Remark 4.9. Notice that there is no closed formula for $A_{a, b}^{R_{p}(t)}$ with $t \in \mathbb{N}^{*}, a, b \in \mathbb{Z}$ and $p \in \mathbb{P}_{k}^{1}$ of degree $d_{p}>1$. This because $A_{a, a}^{R_{p}(t)}=1$ only for $0 \leq a \leq d_{p} t$ with $d_{p} \mid a$. However this case will not appear over $\mathbb{C}$.

\section{A recursive algorithm for the cardinalities $A_{a, b}^{M}=\left|G r_{(a, b)}(M)\right|$ with $M$ arbitrary}

Let $M \in \bmod -k K$ arbitrary and suppose $\underline{\operatorname{dim}} M=(m, n)$. We know that up to isomorphism $M=P \oplus R \oplus I$ where $P$ (respectively $I, R$ ) is a module with all its indecomposable components preprojective (respectively preinjective, regular). We also know that $A_{a, b}^{M}=0$ for $a<0$ or $b<0$ or $a>m$ or $b>n$.

Applying the recursion from Theorem 3.2. a) after a finite number of steps $A_{a, b}^{P \oplus R \oplus I}$ is reduced to knowing some numbers of the form $A_{a^{\prime}, b^{\prime}}^{R^{\prime} \oplus I^{\prime}}$. Applying the recursion from Theorem 3.2. b) after a finite number of steps $A_{a^{\prime}, b^{\prime}}^{R^{\prime} \oplus I^{\prime}}$ is reduced to knowing some numbers of the form $A_{a^{\prime \prime}, b^{\prime \prime}}^{R^{\prime \prime}}$. Using the arguments from the proof of Lemma 4.5. b) we can see that $A_{a^{\prime \prime}, b^{\prime \prime}}^{R^{\prime \prime}}=0$ for $a^{\prime \prime}<b^{\prime \prime}$ so applying the recursion from Theorem 3.2. a) after a finite number of steps $A_{a^{\prime \prime}, b^{\prime \prime}}^{R^{\prime \prime}}$ with $a^{\prime \prime} \geq b^{\prime \prime} \geq 0$ is reduced to knowing some numbers of the form $A_{a^{\prime \prime \prime}, a^{\prime \prime \prime}}^{R^{\prime \prime \prime}}$. (Here "some" means of course "a finite number").

Suppose $R^{\prime \prime \prime}=\oplus_{i=1}^{m} R_{p_{i}}\left(\lambda^{i}\right)$, where $\lambda^{i}$ are partitions, $p_{i} \in \mathbb{P}_{k}^{1}$ different points with degree $d_{p_{i}}$ and $\sum_{i=1}^{m} d_{p_{i}}\left|\lambda^{i}\right|=n$ so $\underline{\operatorname{dim}} R^{\prime \prime \prime}=(n, n)$. Denote $a^{\prime \prime \prime}$ simply by $a$ and suppose $0 \leq a \leq n$. For partitions $\lambda, \mu, \nu$ we will denote by $g_{\nu \mu}^{\lambda}\left(q^{d_{p}}\right)=F_{R_{p}(\nu) R_{p}(\mu)}^{R_{p}(\lambda)}$ the classical Hall polynomial (see [6] for details). We know that $g_{\nu \mu}^{\lambda}=g_{\mu \nu}^{\lambda}$ and $g_{\nu \mu}^{\lambda}=0$ unless $|\lambda|=|\mu|+|\nu|$ and $\mu, \nu \subseteq \lambda$.

Using Lemma 1.1. b) and Lemma 1.4. we have that

$$
\left[\oplus_{i=1}^{m} R_{p_{i}}\left(\nu^{i}\right)\right]\left[\oplus_{i=1}^{m} R_{p_{i}}\left(\mu^{i}\right)\right]=\prod_{i=1}^{m}\left[R_{p_{i}}\left(\nu^{i}\right)\right]\left[R_{p_{i}}\left(\mu^{i}\right)\right]
$$

so

$$
F_{\oplus_{i=1}^{m} R_{p_{i}}\left(\nu^{i}\right) \oplus_{i=1}^{m} R_{p_{i}}\left(\mu^{i}\right)}^{\oplus_{m}^{m} R_{p_{i}}\left(\lambda^{i}\right)}=\prod_{i=1}^{m} F_{R_{p_{i}}\left(\nu^{i}\right) R_{p_{i}}\left(\mu^{i}\right)}^{R_{p_{i}}\left(\lambda^{i}\right)}
$$


Using the considerations above and the arguments from the proof of Lemma 4.5. a) we will have

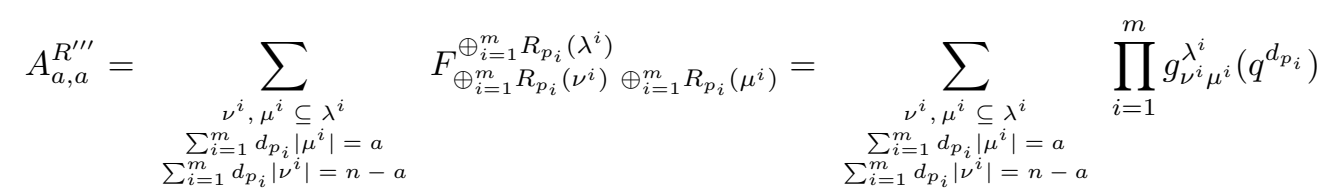

\section{REFERENCES}

[1] I. Assem, D. Simson, A. Skowronski, Elements of Representation Theory of Associative Algebras, Volume 1: Techniques of Representation Theory. LMS Student Texts (No. 65) (Cambridge Univ. Press 2006).

[2] M. Auslander, I. Reiten, S. Smalo, Representation Theory of Artin Algebras, Cambridge Stud. in Adv. Math. 36 (Cambridge Univ. Press 1995).

[3] P. Caldero, M. Reineke, On the quiver Grassmannians in the acyclic case. Preprint arxiv math.RT/0611074

[4] P. Caldero, A. Zelevinsky, Laurent expansions in cluster algebras via quiver representations. Moscow Mathematical Journal, Vol. 6, special issue in honor of Alexander Alexandrovich Kirillov on the occasion of his seventieth birthday, (2006), 411-429.

[5] V. Dlab, C. M. Ringel, Indecomposable representations of graphs and algebras. AMS Memoirs 173 (1976).

[6] I. G. Macdonald, Symmetric Functions and Hall Polynomials. Clarendon Press Oxford 1995.

[7] T. S. Nanjundiah, Remark on a note of P. Turán. Am. Monthly 65(1958), 354.

[8] C. M. Ringel, Tame algebras and Integral Quadratic Forms. Lect. Notes Math. 1099 (Springer 1984).

[9] Cs. Szántó, Hall numbers and the composition algebra of the Kronecker algebra. Algebras and Representation Theory 9,(2006), 465-495. 